\title{
Correlation Between Expressions of VEGF and CA-125 with Stage, Level of Differentiation and Histopathology Type of Epithelial Ovarian Cancer
} \author{
Upik A. Miskad ${ }^{3}$ \\ ${ }^{1}$ Department of Obstetric and Gynecology, Medical Faculty, Hasanuddin University, Makassar, Indonesia \\ ${ }^{2}$ Department of Biostatistic, Faculty of Public Health, Hasanuddin University, Makassar, Indonesia \\ ${ }^{3}$ Department of Anatomical Pathology, Medical Faculty, Hasanuddin University, Makassar, Indonesia
}

Farid Abdullah $^{1}$, Syahrul Rauf ${ }^{1}$, Nusratuddin Abdullah ${ }^{1}$, Maisuri T. Chalid ${ }^{1,2}$, Deviana S. Riu ${ }^{1}$,

Email address:

farid_encephalon@yahoo.com (F. Abdullah)

\section{To cite this article:}

Farid Abdullah, Syahrul Rauf, Nusratuddin Abdullah, Maisuri T. Chalid, Deviana S. Riu, Upik A. Miskad. Correlation Between Expressions of VEGF and CA-125 with Stage, Level of Differentiation and Histopathology Type of Epithelial Ovarian Cancer. Journal of Gynecology and Obstetrics. Vol. 4, No. 6, 2016, pp. 62-67. doi: 10.11648/j.jgo.20160406.16

Received: December 4, 2016; Accepted: December 15, 2016; Published: January 10, 2017

\begin{abstract}
Cancer Antigen (CA-125) is a tumor marker used to detect ovarian cancer. This study aims to determine the relationship of VEGF expression and the levels of CA-125 on primary tumor associated with stage, degree of differentiation and histopathological types of epithelial ovarian cancer. This study is an observational study with a cross sectional design in Wahidin Sudirohusodo Hospital and some parts teaching hospital of Obstetrics and Gynecology began in August 2015 to February 2016. The population was all patients with epithelial ovarian cancer who were treated and underwent surgery at the hospital who met the inclusion criteria. The data were processed using SPSS with bivariate analysis Mann Whitney, Kruskal Wallis and Spearman's Rho. The result there were significant differences of VEGF expression in cell differentiation and histopathological type $(\mathrm{p}<0.05)$. There are significant differences in the levels of CA-125 with clinical stage, differentiation of cells and histopathological type $(\mathrm{p}<0.05)$. The expression of VEGF is getting stronger when the type of epithelial ovarian cancer is getting bad influence on the degree of cells differentiation and the histopathological type. CA-125 levels obtained increased at an advanced stage and degree of cells differentiation are bad for ovarian cancer. There is a positive correlation between the expression of VEGF and CA-125 levels in epithelial ovarian cancer but was not significantly different and the relationship is weak.
\end{abstract}

Keywords: VEGF, CA-125, Epithelial Ovarian Cancer

\section{Introduction}

Ovarian cancer is the most deadly gynecology malignancy. General Pathophysiology for ovarian cancer is peritoneal metastasis, and ascites accumulation often happened progressively with or without malignancy cells in peritoneal fluid. Metastasis to peritoneal cavity is connected with several non-specific symptoms which often misdiagnosed with gastro-intestine or other internal genitalia diseases. Some generally found symptoms are the abdominal discomfort and full sensation. Other problem found is difficulties to palpate the ovary mass which still in pelvic cavity. Because of these reason (non-specific symptom and un-palpable mass), around $70 \%$ patients are late diagnosed at stage III or IV $[1,2,3,4]$.

Around 90 to $95 \%$ cases of ovarian cancer are consisting of epithelial ovary carcinoma. Epithelial ovarian cancer management is based on combination of surgery and chemotherapy. Tumor mass debulking and followed with platinum based chemotherapy is considered as treatment standard for late stage of epithelial ovary carcinoma. Although aggressive primary treatment is giving good 
respond rate, most of late stage epithelial ovarian cancer patient will experience cancer relapse and chemotherapy resistance. It shows the demand for new strategy and better therapy agent $[1,3]$.

Tumor growth and metastasis is fully depending on vascularization makes angiogenesis therapy to be rational therapy agent. Angiogenesis itself is the primary sign for wound healing, menstruation cycle, cancer, various ischemia, and inflammation diseases. When the vascular shrink to 2.0 $\mathrm{mm}$ in diameter, the tumor is begin to hypoxia and begin to stimulating angiogenesis process. Various pro- and antiangiogenic are found. Vascular Endothelial Growth Factor (VEGF) is angiogenesis and lymph-angiogenesis stimulant which interested and many studied. Signaling transduction includes the bonding to thyrosine kinase and initiating endothelial cell proliferation, migration, and building new vascularization [2].

Vascular Endothelial Growth Factor also known as Vascular Permeability Factor, multifunctional cytokine which increasing microvascular permeability and directly stimulating endothelial cells and angiogenesis, with bending to VEGF specific receptor at vascular endothelial cells. Vascular Endothelial Growth Factor synthesized and excreted by various human's cultured cancer cells and solid cancer, such as brain cancer, lung cancer, breast cancer, and ovarian cancer, also digestive, kidney, and vesicaurinaria adenocarcinoma. VEGF increased expression is considered involved in carcinogenesis, metastasis, and effusion production by increasing vascular permeability or angiogenesis, so there are many studies which support anti VEGF using [2, 5].

CA-125 is one of tumor marker used for detecting ovarian cancer. CA-125 is newly found at 1981, and in this last 5 years, CA-125 to be first choice for detecting ovarian cancer. CA-125 is glycoprotein at cells surface with high molecule weight (more than $1000 \mathrm{kDa}$ ) [6].

Previous study by Gorp et al. 2011, Suh et al. 2012 are found increasing CA-125 concentration at woman with ovarian cancer $(82 \%)$ compared with healthy woman $(1 \%)$, at benign tumor $(6 \%)$, and non gynecological cancer $(28.5 \%)$. However, CA-125 is not specific marker because it can give fairly big number of false positive, where CA-125 also increased in several other cases such as: endometriosis, fibroid, benign ovary cyst, pelvic infection, pregnancy, menstruation, liver diseases, and inflammation diseases. CA125 concentration is increased roughly $83 \%$ for type epithelial ovarian cancer, but keeps low for mucinous type. $[7,8]$

Other studies by Sarojin et al. 2012, Suh et al. 2012, and Rancouert et al 2012 are found CA-125 concentration is increased $25-30 \%$ at ovarian cancer stage I and around $90 \%$ at late stage, so CA-125 is very usable for detecting late stage ovarian cancer, but we still need to find another biomarker with highest sensitivity and specificity for detecting early stage epithelial ovarian cancer to increasing the overall survivability of ovarian cancer patients. $[6,8,9]$

Until now, we still searching for very sensitive and specific biomarker for establish early diagnose of ovarian cancer patient and then reducing their morbidity and mortality. Relation of Vascular Endothelial Growth Factor expression and concentration of Cancer Antigen 125 (CA-125) for epithelial ovarian cancer is not yet published.

Based on background explanation, Author interested to do a study which aimed to knowing the relation of Vascular Endothelial Growth Factor expression and CA-125 concentration at primary cancer based on stage, differentiation degree, and histopathology type of epithelial ovarian cancer.

\section{Material and Methods}

\subsection{Location and Study Period}

This study is held at Obstetrics-Gynecology department of several educational hospitals of Medical Faculty Hasanuddin University, such as: Dr. Wahidin Sudirohusodo Hospital, Pelamonia Hospital, Labuang Baji Public Hospital, Faisal Islamic Hospital, Bhayangkara Hospital, IbnuSina Hospital and Syekh Yusuf Regional Public Hospital. This study is held after obtaining the permission of regional authorities and patient for sampling. This study period is begin at August 2015 and end at February 2016.

\subsection{Design and Research Variables}

This study is observational study with cross sectional design, aimed for knowing the relation between VEGF expression and CA-125 concentration at epithelial type ovarian cancer.

\subsection{Population and Sample}

Population in this study is all solid/cystic ovary neoplasm with suspected malignant who treated and going for surgery at obstetrics-gynecology department of Medicine Faculty Hasanuddin University of Dr. Wahidin Sudirohusodo Hospital or other hospitals as mentioned above, which used for educational place of obstetrics-gynecology department Medicine Faculty Hasanuddin University. Study sample is some tissue of solid/cystic ovary neoplasm with suspected malignant from patient who going for surgery at educational places of obstetrics-gynecology department of Medicine Faculty Hasanuddin University from August 2015 until at end of study period or until the sample amount is fulfilled.

\subsection{Method of Collecting Data}

All study subject who fill study criteria are included: histopathology type, cell differentiation degree, and stage of ovarian cancer; patient's agreement for included in this study; and to fill the prepared questionnaire which consist of anamneses result, physical examination result, and laboratory result. All prepared sample, instrument, material are used for making tissue sample for microscope examination and VEGF immunohistochemistry and then interpreted. 


\subsection{Data Analysis Technique}

All data obtained and grouped based with the aim and data type, and then processing it with suitable statistic method with SPSS v. 16 software for windows.

(1). Bivariate Analysis Test X2 (Chi-square)

Used to compare values variable the nominal scale of two or more groups that are not in pairs. In this case comparing the expression of VEGF for each type of histopathology and clinical stage epithelial ovarian cancer if Chi Square test requirements is fulfilled.

(2). Mann Whitney Test

Chi square test if the requirements is not fulfilled then the alternative test which can be used when a tables $2 \times \mathrm{K}$, ordinal and objectives can be used to compare trends Mann Whitney test

\section{(3). Kruskal Wallis test}

Is one alternative of Chi Square test if the condition is not fulfilled in the form table $>2 x>2$ and one of the ordinal variables and objectives see the trend, it can be used Kruskal Wallis test.

\section{Result}

We're held an observational study with cross sectional setting for knowing correlation between VEGF expression and CA-125 concentration which connected with stage, differentiation degree, and histopathology type of epithelial ovarian cancer. Several educational hospitals of obstetricsgynecology department medical faculty Hasanuddin University such as: Dr. Wahidin Sudirohusodo Hospital, Pelamonia Hospital, Labuang Baji Public Hospital, Faisal Islamic Hospital, Bhayangkara Hospital, Ibnu Sina Hospital and Syekh Yusuf Regional Public Hospital. This study is begin at August 2015 and ended at February 2016. For this study, we got 18 cases of early stage ovarian cancer and 38 cases of late stage.

Our study results showed that VEGF-0 expression is mostly found at early stage (4 cases, $80 \%$ ), while VEGF-3 expression is mostly found at late stage ( 8 cases, $80 \%$. There is no significant difference between various expression degrees of VEGF among all clinical stage of epithelial ovarian cancer. $(\mathrm{p}>0.05)$ (Table 1$)$.

Table 1. Distribution of expression of VEGF by clinical stadium on epithelial ovarian cancer tissue.

\begin{tabular}{|c|c|c|c|c|c|c|c|}
\hline \multirow{3}{*}{ VEGF Expression } & \multicolumn{4}{|c|}{ Clinical Staging } & & & \\
\hline & \multicolumn{2}{|c|}{ Early stage } & \multicolumn{2}{|c|}{ Late stage } & \multicolumn{2}{|c|}{ Total } & \multirow[t]{2}{*}{$\mathbf{P}$} \\
\hline & $\mathbf{n}$ & $\%$ & $\mathbf{n}$ & $\%$ & $\mathbf{n}$ & $\%$ & \\
\hline 0 & 4 & 80 & 1 & 20 & 5 & 100 & $0.070 *$ \\
\hline 1 & 7 & 31,8 & 15 & 68,2 & 22 & 100 & \\
\hline 2 & 5 & 26,3 & 14 & 73,7 & 19 & 100 & \\
\hline 3 & 2 & 20 & 8 & 80 & 10 & 100 & \\
\hline Total & 18 & 32,1 & 38 & 67,9 & 56 & 100 & \\
\hline
\end{tabular}

*Mann Whitney test

Based on cell differentiation status, VEGF-0 expression all means well differentiation for $100 \%$ of well differentiated cancer patients, while VEGF-3 expression means most of them poor differentiated, for $70 \%$ of patients. Result of Kruskal Wallis statistical test showing there is significant relation between VEGF expression with cell differentiation of epithelial ovarian cancer tissue $(\mathrm{p}<0.05)($ Table 2$)$.

Table 2. Distribution of VEGF expression by cell differentiation on epithelial ovarian cancer tissue.

\begin{tabular}{|c|c|c|c|c|c|c|c|c|c|}
\hline \multirow[b]{2}{*}{ VEGF Expression } & \multicolumn{6}{|c|}{ Cell differentiation } & \multirow{2}{*}{\multicolumn{2}{|c|}{ Total }} & \multirow[b]{2}{*}{$\mathbf{P}$} \\
\hline & \multicolumn{2}{|c|}{ Well } & \multicolumn{2}{|c|}{ Moderate } & \multicolumn{2}{|c|}{ Poor } & & & \\
\hline 0 & 5 & 100 & 0 & 0 & 0 & 0 & 5 & 100 & $0.001 *$ \\
\hline 1 & 7 & 31,8 & 11 & 50 & 4 & 18,2 & 22 & 100 & \\
\hline 2 & 2 & 10,5 & 12 & 63,2 & 5 & 26,3 & 19 & 100 & \\
\hline 3 & 2 & 20 & 1 & 10 & 7 & 70 & 10 & 100 & \\
\hline Total & 16 & 28,6 & 24 & 42,9 & 16 & 28,6 & 56 & 100 & \\
\hline
\end{tabular}

*Kruskal Wallis test

VEGF-0 expression is mostly found at mucinous histopathology type (80\%), while VEGF-3 is mostly found at serous type (90\%). Result of Kruskal Wallis statistical test showing there is significant relation between VEGF expression with histopathology type of epithelial ovarian cancer tissue $(\mathrm{p}<0.05)$ (Table 3$)$. 
Table 3. Distribution of VEGF expression by Histopathology type on epithelial ovarian cancer tissue.

\begin{tabular}{|c|c|c|c|c|c|c|c|c|c|c|c|}
\hline \multirow{3}{*}{ VEGF Expression } & \multicolumn{8}{|c|}{ Histopathology type } & \multirow{2}{*}{\multicolumn{2}{|c|}{ Total }} & \multirow{3}{*}{$\mathbf{P}$} \\
\hline & \multicolumn{2}{|c|}{ Mucinous } & \multicolumn{2}{|c|}{ Serous } & \multicolumn{2}{|c|}{ Endometrioid } & \multicolumn{2}{|c|}{ Clear cell } & & & \\
\hline & $\mathrm{n}$ & $\%$ & $\mathrm{n}$ & $\%$ & $\mathbf{N}$ & $\%$ & $\mathrm{n}$ & $\%$ & $\mathrm{n}$ & $\%$ & \\
\hline 0 & 4 & 80 & 1 & 20 & 0 & 0 & 0 & 0 & 24 & 100 & $0.028^{*}$ \\
\hline 1 & 9 & 40,9 & 8 & 36,4 & 5 & 22,7 & 0 & 0 & 22 & 100 & \\
\hline 2 & 4 & 21,1 & 13 & 68,4 & 1 & 5,3 & 1 & 5,3 & 19 & 100 & \\
\hline 3 & 1 & 10 & 9 & 90 & 0 & 0 & 0 & 0 & 10 & 100 & \\
\hline Total & 18 & 32,1 & 31 & 55,4 & 6 & 10,7 & 1 & 1,8 & 56 & 100 & \\
\hline
\end{tabular}

* Kruskal Wallis test

CA-125 concentration $\leq 35$ is mostly found at early stage (62.5\%), CA-125 concentration 36 to 600 and $>600$ is mostly found at late stage $(58.3 \%$ and $87.5 \%$ in a row). Statistical Mann Whitney test result showing there is significant relation between CA-125 concentration and clinical staging of epithelial type ovarian cancer $(p<0.05)($ Table 4$)$.

Table 4. Distribution of CA-125 Concentration by clinical stadium in epithelial ovarian cancer tissue.

\begin{tabular}{|c|c|c|c|c|c|c|c|}
\hline \multirow{3}{*}{$\begin{array}{l}\text { CA-125 } \\
\text { Concentration }\end{array}$} & \multicolumn{4}{|c|}{ Clinical Staging } & \multirow{2}{*}{\multicolumn{2}{|c|}{ Total }} & \multirow{3}{*}{$\mathbf{P}$} \\
\hline & \multicolumn{2}{|c|}{ Early Stage } & \multicolumn{2}{|c|}{ Late Stage } & & & \\
\hline & $\mathbf{n}$ & $\%$ & $\mathbf{n}$ & $\%$ & $\mathbf{n}$ & $\%$ & \\
\hline$\leq 35$ & 5 & 62,5 & 3 & 37,5 & 8 & 100 & $0.004 *$ \\
\hline $36-600$ & 10 & 41,7 & 14 & 58,3 & 24 & 100 & \\
\hline$>600$ & 3 & 12,5 & 21 & 87,5 & 24 & 100 & \\
\hline Total & 18 & 32,1 & 38 & 67,9 & 56 & 100 & \\
\hline
\end{tabular}

*Mann Whitney test

CA-125 concentration $\leq 35$ and 36 to 600 are mostly found at good differentiating epithelial type ovarian cancer (62.5\% and $41.7 \%$ in a row), while CA-125 concentration $>600$ is mostly found at badly differentiated (54.2\%). Statistical Kruskal Wallis test result showing there is significant relation between CA-125 concentration and cell differentiation degree of epithelial type ovarian cancer $(\mathrm{p}<0.05)$ (Table 5).

Table 5. Distribution of CA-125 Concentration bycell differentiation in epithelial ovarian cancer tissue.

\begin{tabular}{|c|c|c|c|c|c|c|c|c|c|}
\hline \multirow{3}{*}{$\begin{array}{l}\text { CA-125 } \\
\text { Concentration }\end{array}$} & \multicolumn{6}{|c|}{ Cell differentiation } & \multirow{2}{*}{\multicolumn{2}{|c|}{ Total }} & \multirow{3}{*}{$\mathbf{P}$} \\
\hline & \multicolumn{2}{|c|}{ Well } & \multicolumn{2}{|c|}{ Moderate } & \multicolumn{2}{|c|}{ Poor } & & & \\
\hline & $\mathbf{n}$ & $\%$ & $\mathbf{n}$ & $\%$ & $\mathbf{n}$ & $\%$ & $\mathbf{n}$ & $\%$ & \\
\hline$\leq 35$ & 5 & 62,5 & 2 & 25 & 1 & 12,5 & 8 & 100 & $0.001 *$ \\
\hline $36-600$ & 10 & 41,7 & 9 & 37,5 & 5 & 20,8 & 24 & 100 & \\
\hline$>600$ & 1 & 4,2 & 10 & 41,7 & 13 & 54,2 & 24 & 100 & \\
\hline
\end{tabular}

*Kruskal Wallis test

CA-125 concentration $\leq 35$ is mostly found at mucinous type $(62.5 \%)$, while CA-125 concentration 36 to 600 is mostly found at both mucinous and serous type (41.7\%) and CA-125 concentration $>600$ is mostly found at serous type (75\%). Statistical Kruskal Wallis test result showing there is significant relation between CA-125 concentration and histopathology type of epithelial type ovarian cancer tissue. $(\mathrm{p}<0.05)$ (Table 6$)$.

Table 6. Distribution of CA-125 Concentration by histopathology type in epithelial ovarian cancer tissue.

\begin{tabular}{|c|c|c|c|c|c|c|c|c|c|c|c|}
\hline \multirow{3}{*}{$\begin{array}{l}\text { CA-125 } \\
\text { Concentration }\end{array}$} & \multicolumn{8}{|c|}{ Histopathology type } & \multirow{2}{*}{\multicolumn{2}{|c|}{ Total }} & \multirow{3}{*}{$\mathbf{P}$} \\
\hline & \multicolumn{2}{|c|}{ Mucinous } & \multicolumn{2}{|c|}{ Serous } & \multicolumn{2}{|c|}{ Endometrioid } & \multicolumn{2}{|c|}{ Clear cell } & & & \\
\hline & $\mathbf{n}$ & $\%$ & $\mathbf{n}$ & $\%$ & $\mathbf{N}$ & $\%$ & $\mathbf{n}$ & $\%$ & $\mathbf{n}$ & $\%$ & \\
\hline$\leq 35$ & 5 & 62,5 & 3 & 37,5 & 0 & 0 & 0 & 0 & 8 & 100 & $0.028 *$ \\
\hline $36-600$ & 10 & 41,7 & 10 & 41,7 & 3 & 12,5 & 1 & 4,2 & 24 & 100 & \\
\hline$>600$ & 3 & 12,5 & 18 & 75 & 3 & 12,5 & 0 & 0 & 24 & 100 & \\
\hline Total & 18 & 32,1 & 31 & 55,4 & 6 & 10,7 & 1 & 1,8 & 56 & 100 & \\
\hline
\end{tabular}

*Kruskal Wallis test 
At correlation Spearman's rho test for correlation between VEGF and CA-125 concentration for epithelial ovarian cancer, we got $\mathrm{p}=0.067$ means there is insignificant relation with $r=0.247$, means there is positive correlation but weak.

\section{Discussion}

This study showing there is significant VEGF expression difference related with cell differentiation and histopathology type $(\mathrm{p}<0.05)$ and there is significant CA-125 concentration difference related with clinical staging, cell differentiation and histopathology type $(\mathrm{p}<0.05)$.

Vascular Endothelial Growth Factor (VEGF) is proofed to have big influence for forming new vascular to cancer, to supply food for cancer with high metabolism rate, and to prepare access to Host's vascular system. Several studies showing there is VEGF have influence for various ovary carcinogenesis stages, with influencing cancer growth rate and to forming new vascular as seen at animal and human model. Olson et al. (2008) is the first study about VEGF genetic expression at normal and cancerous human ovaries, with result that VEGF is important mediator for forming ascites and cancer metastatic of cancerous ovary. [10]

VEGF is causing angiogenesis greatly that enable the cancer to growth exponentially, also enable the cancer to spread to faraway places as metastatic cancer. Several studies publish there is positive relation between VEGF expression and stage of ovarian cancer.

This study is not getting significant relation between VEGF expression and stage of ovarian cancer, but the different result is showed at Smerdel et al. studies that showing there is significant relation between VEGF expression and stage of ovarian cancer. This different result is probably caused of different technique test used. At Smerdel's study, they are testing VEGF concentration and VEGF genetic expression with ELISA method, while at our study, we using immunohistochemical method. Several studies already confirm that result of ELISA and immunohistochemical method is not always same. [11]

At this study, we are getting significant difference of VEGF expression and differentiation degree. The similar result is showed at study by $\mathrm{Yu}$ et al that found stronger VEGF expression at epithelial type ovarian cancer means higher differentiation degree and worsens prognostics.[12]

This significant correlation degree is proofing that theory about changing of VEGF sub-cellular distribution is following progression change to worsen of ovary carcinoma is true indeed, with correlation of increased VEGF expression is followed by higher cancer cell differentiation, which giving clue that excess of VEGF expression is related with beginning, recurrent, and prognostic of cancer diseases. This result is similar with many studies reporting that amount of VEGF production rate at cancer tissue is related with cancer growth rate and disadvantage clinicopathologic variables, such as bad prognosis, short life span, and chemotherapy resistant. Increasing in cancer cell differentiation is linearly related with increasing in VEGF expression. Higher VEGF expression is related with worse differentiation of ovarian cancer cell. $[12,13]$

At this study, we are getting significant relation between VEGF expression and histopathology type. It's different with study by Secord et al and Yu et al (2013) which got insignificant relation. However, both studies are getting most of them was serous histopathology type, as our study got. At our study, we can see the tendency for VEGF expression increased for serous histopathologic type. $[12,14]$

The previous understanding that says ovary carcinogenesis for various cancer type are all originated from epithelial ovary surface (mesothelium cell) and because of metaplastic change, the cell also change to various type (Serous, Endometrioid, Clear Cell, Mucinous, and Transitional Cell), but new understanding says that ovarian cancer have probability to also originated from Tuba and Endometrium. [15]

CA-125 is tumor marker which used for detecting ovarian cancer. CA-125 concentration's role is as oncogene which increasing cancer cell's motility, migration, and invasion of ovarian cancer. At this study, CA-125 at early stage and late stage are compared and we found significant difference ( $p$ $<0.05$ ). That result is appropriate with result of study by Hogdal et al (2007), which found that CA-125 at cancer tissue is closely related with ovarian cancer clinical staging (FIGO) and related with bad prognosis. The result also appropriate with theory that says ovary carcinoma is biggest cause of the death among all kind of genitalia cancer, and placed at $4^{\text {th }}$ biggest cause of death among all kind of cancer at Europe and America. At Indonesia, ovary carcinoma is placed at $6^{\text {th }}$ after cervix carcinoma, breast cancer, colorectal cancer, skin cancer, and lymphoma. From Indonesian studies, we got incidence of ovary carcinoma around 10 to $13 \%$ from all gynecology carcinomas. Patient of ovary carcinoma usually lately diagnosed, and only 20 to $35 \%$ among them diagnosed at early stage. That's because early stage of ovary carcinoma is asymptomatic, and usually around $75 \%$ ovary carcinoma will detected at late stage because of belly enlargement, ascites, colon partial obstruction, and also finding metastases to surrounding organs, lymph nodes, or at far away. These makes bad prognosis of ovary carcinoma, because surgery therapy and chemotherapy at late stage have low survival rate. [16]

We got significant result of CA-125 concentration ( $p$ $<0.05$ ) in various differentiation degree. This similar result is also published by Cambruzi E et al (2014), which found that high CA-125 concentration at medium and high differentiation degree. This finding is related with higher CA125 concentration at worse differentiation degree, and not only showing how malignant the ovarian cancer is, but also related with cancer's high growth rate. More necrosis areas mean higher ovarian cancer progressivity. [16]

We got significant result of CA-125 concentration in different histopathology type. This result is not found at study by Rao et al, Liu et al, and Hogdal et al which says no 
correlation between histopathology type and CA-125 concentration. At this study, most sample have CA-125 concentration above $35 \mathrm{U} / \mathrm{mL}$ at all histology type of ovarian cancer. Statistically, we found significant difference in histology type, which we mostly found serous type 31 cases (55.4\%), mucinous 18 cases $(32.1 \%)$, endometrioid 6 cases $(10.7 \%)$ and clear cell 1 case $(1.8 \%)$. This similar result is also published by Hala A et al (2010), where they got serous, mucinous, and endometroid type at $66.7 \%, 25 \%$, and $8.3 \%$; Gorp TV et al (2011) found serous type 84 cases (52.2\%) of 131 epithelial type ovary carcinoma cases; and Elham O A et al (2013) found serous type 21 cases $(70 \%)$ of 30 epithelial type ovary carcinoma. Serous histology type of epithelial ovary cardinoma is the most often found because several reason. Mccluggage G (2007) says about serous adenocarcinoma is the most often found type of epithelial ovary carcinoma, and most of serous type of epithelial ovary carcinoma is found at late stage (Stage III \& IV), and our study shows similar result, where most of our 31 cases of serous type at late stage. Study by Levanon K et al (2008) published that epithelial ovary carcinoma's origin from several places: ovary (for serous ovary carcinoma), endosalphynx (for serous tuba fallopii carcinoma) and peritoneum (for serous primary peritoneum carcinoma). This is one reason for why serous type epithelial ovary carcinoma is the most often found. [17]

We conclude that there is insignificant positive relation between VEGF relation and CA-125 concentration at epithelial ovarian cancer, but it have weak correlation coefficient. We also found VEGF and CA-125 involvement with ovarian cancer progressivity.

\section{Conclusion and Suggestion}

The author of this study conclude that stronger VEGF expression for epithelial ovarian cancer means increased in stadium, worse differentiation degree, and more probability for serous type. CA-125 concentration is increased at late stage, worse differentiation degree, and even higher for serous type. There is positive correlation between VEGF expression and CA-125 concentration but weak and insignificant. The author suggest to do more advance study, especially to value relation of VEGF expression in order to reach better treatment strategy for ovary malignancies. More investigation needed for knowing more about involvement of VEGF and CA-125 in ovarian cancer progressivity.

\section{References}

[1] Lengyel. (2010). Ovarian Cancer Development and Metastasis. The American Journal of Pathology, Vol. 177, No. 3.

[2] Yamamoto et al. (2007). Expression of Vascular Endothelial
Growth Factor (VEGF) in Epithelial Ovarian Neoplasms: Correlation With Clinicopathology and Patient Survival, and Analysis of Serum VEGF Levels. British Journal of Cancer 76 (9), 1221-1227.

[3] Spannuth et al. (2008). Angiogenesis as a Strategic Target for Ovarian Cancer Therapy. Nature Clinical Practice Oncology. Vol 5 no 4.

[4] Gubbels et al. (2010). The Detection, Treatment, and Biology of Epithelial Ovarian Cancer. Journal of Ovarian Research., 3: 8

[5] Amini et al. (2012). Utility of Vascular Endothelial Growth Factor Inhibitors in the Treatment of Ovarian Cancer: From Concept to Application. Hindawi Publishing Corporation. Journal of Oncology.

[6] Rancouert et al. (2012). The Role of MUC 16 Mucin (CA 125) in the Pathogenesis of Ovarian Cancer. Intechopen.

[7] Gorp et al. (2011). HE4 and CA-125 as a Diagnostic Test in Ovarian Cancer: Prospective Validation of The Risk of Ovarian Malignancy Algorithm. British Journal of Cancer.

[8] Suh et al. (2012). Ovarian Cancer Biomarkers For Molecular Biosensors And Translational Medicine. Expert-Riview, 10, 1069-1083.

[9] Sarojin et al. (2012). Early Detection Biomarker For Ovarian Cancer. Journal of oncology, 1-15.

[10] Olson et al. (2008). Vascular Permeability Gene in Normal and Neoplastic Human Ovaries. Cancer research, 74, 276-80.

[11] Smerdel et al. (2011). VEGF in the Development of Ovarian Malignancy. Clinical Ovarian Cancer, 4, 19-25.

[12] Yu et al. (2013). The Prognostic Value of Vascular Endothelial Growth Factor in Ovarian Cancer; A Systematic Review and Meta Analysis. Gynecologic Oncology, 128. 391-396.

[13] Landen et al. (2008). Early Events in The Pathogenesis of Epithelial Ovarian Cancer. J clint Oncol., 26. 995-1005.

[14] Secord et al. (2007). Co-expression of Angiogenic Markers and Associations With Prognosis in Advanced Epithelial Ovarian Cancer: A Gynecologic Oncology Group Study. Gynecologic Oncology, 105, 221-232.

[15] Kurman et al. (2008). Early Detection and Treatment of Ovarian Cancer: Shifting From Early Stage to Minimal Volume of Disease Based on a New Model of Carcinogenesis. Am J Obstet Gynecol, 198, 351-356.

[16] Colombo \& Parma. (2006). Ovarian Cancer. European Institute of Oncology, Division of Gynecology, Ripamonti: Milan, Italy University Hospitals Leuven, Belgium, Istituto Nazionale per lo Studlo ela Curadei Tumori, Milan, Italy Istituto Oncologi codella Svizzera Italiana (IOSI), Bellinzona, Switzeland.

[17] Hogdall et al. (2007). CA125 Expression Pattern, Prognosis and Correlation With Serum CA125 in Ovarian Tumor Patients From the Danish "MALOVA" Ovarian Cancer Study. Gynecol Oncol. 104: 508-515. 